\title{
Overexpression of microRNA-24 increases the sensitivity to paclitaxel in drug-resistant breast carcinoma cell lines via targeting $\mathrm{ABCB9}$
}

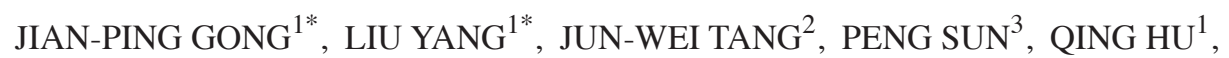 \\ JIAN-WEI QIN $^{1}$, XIAO-MING XU ${ }^{1}$, BEI-CHENG SUN ${ }^{2}$ and JIN-HAI TANG ${ }^{1}$ \\ ${ }^{1}$ Department of General Surgery and Breast Cancer Center, The Affiliated Cancer Hospital of \\ Nanjing Medical University \& Jiangsu Institute of Cancer Research; ${ }^{2}$ Liver Transplantation Center of \\ The First Affiliated Hospital and Cancer Center, Nanjing Medical University, Nanjing, Jiangsu 210009; \\ ${ }^{3}$ School of Pharmacy, Shanghai University of Traditional Chinese Medicine, Shanghai 201203, P.R. China
}

Received June 5, 2015; Accepted August 25, 2016

DOI: $10.3892 / 01.2016 .5139$

\begin{abstract}
Paclitaxel has been widely used in the treatment of breast cancer. However, the development of drug resistance often increases the failure of chemotherapy. Growing evidence has reported the significant role of microRNAs (miRs) in drug resistance. The present study identified that miR-24 was significantly downregulated in paclitaxel-resistant (PR) breast cancer patients and in MCF-7/PR human breast carcinoma cells, and that overexpression of miR-24 could increase the effect of paclitaxel on drug-resistant breast carcinoma cells. Furthermore, miR-24 could directly bind to the 3'-untranslated region of ATP binding cassette $\mathrm{B} 9$ to downregulate its expression, thereby reducing drug transportation and improving the anti-tumor effect of paclitaxel on breast cancer cells. In vivo experiments also demonstrated that overexpression of miR-24 could increase the sensitivity of drug-resistant MCF-7 cells to paclitaxel. In conclusion, the present results suggested a novel function for miR-24 in reducing paclitaxel resistance in breast cancer, which may be of important clinical significance.
\end{abstract}

Correspondence to: Dr Bei-Cheng Sun, Liver Transplantation Center of The First Affiliated Hospital and Cancer Center, Nanjing Medical University, 42 Baiziting Road, Nanjing, Jiangsu 210009, P.R. China

E-mail:sunbc@njmu.edu.cn

Dr Jin-Hai Tang, Department of General Surgery and Breast Cancer Center, The Affiliated Cancer Hospital of Nanjing Medical University \& Jiangsu Institute of Cancer Research, 42 Baiziting Road, Nanjing, Jiangsu 210009, P.R. China

E-mail: jinhai-tang@sohu.com

${ }^{*}$ Contributed equally

Key words: breast cancer, paclitaxel resistance, miR-24, ABCB9

\section{Introduction}

Breast cancer is one of the most common cancers in women, and is the leading cause of cancer-induced mortality (1). With the development of therapeutic methods, including surgical resection, radiotherapy and particularly chemotherapy, the outcomes in breast cancer patients have improved in the last decades $(2,3)$. Multiple anti-cancer drugs have been developed for chemotherapy in cancer therapy. As a natural compound isolated from the bark of the Taxus baccata plant, paclitaxel could bind to the $\beta$-tubulin subunit of microtubules, thereby stabilizing it and forcing dividing cancer cells to arrest in the M phase of the cell cycle, thus promoting cell death (4). Paclitaxel has been successfully used in clinical treatment for $>2$ decades, which significantly improved the outcomes of breast cancer patients (4).

However, acquired drug resistance during treatment occurs in numerous patients and contributes to $90 \%$ of the chemotherapy failures (5). Thus, to explore how cancer cells can eliminate the damage effect of chemotherapeutic drugs such as paclitaxel and improve drug sensitivity could be important for the treatment of cancers. A crucial cause of drug resistance may be the increased expression of efflux pumps (6). Certain transmembrane proteins could transport drugs out of cancer cells. It has been reported that human transporter associated with antigen processing-like, also known as ATP binding cassette $(\mathrm{ABC}) \mathrm{B}$, and other members of the $\mathrm{ABC}$ transporter family could mainly contribute to paclitaxel resistance $(7,8)$. In addition, increased metabolism of chemotherapeutic agents by cytochrome $\mathrm{p} 450$, various $\beta$-tubulin mutations and other mechanisms could together contribute to the resistance to paclitaxel and other chemotherapeutic agents $(9,10)$. Therefore, reducing drug resistance may improve the treatment of breast and other cancers.

MicroRNAs (miRs) are a class of small non-coding RNAs that can control the expression of multiple genes via binding to their 3'-untranslated region (UTR) and consequently inducing post-transcriptional repression or degradation of the messenger RNAs (mRNAs) (11). Recent studies have reported 
that microRNAs are important in drug resistance $(12,13)$. For example, miR-29 could modulate collagen type I alpha 1 expression to improve cisplatin-induced cytotoxicity in ovarian cancer cells (14), and miR-301a increased doxorubicin resistance in osteosarcoma cells by regulating AMP-activated protein kinase $\alpha 1$ (15). In addition, paclitaxel resistance could be also regulated by miRs (16).

miR-24 has been widely reported to be involved in multiple cancers and to mediate tumor growth and apoptosis $(17,18)$. However, the role of miR-24 in cancer is complex and remains unclear. In the present study, it was observed that miR-24 was significantly decreased in paclitaxel-resistant (PR) MCF-7 human breast cancer cells. To further investigate the role of miR-24 in drug resistance of breast cancer, MCF-7/PR cells were established, and the effect of miR-24 on the sensitivity to paclitaxel of MCF-7/PR cells was studied both in vitro and in vivo.

\section{Materials and methods}

Cell culture and establishment of PR cell lines. MCF-7 and SKBR3 human breast cancer cells were obtained from the American Type Culture Collection (Manassas, VA, USA). Cells were cultured in Dulbecco's modified Eagle medium (containing $25 \mathrm{mM}$ glucose and 10\% fetal bovine serum) (GE Healthcare Life Sciences HyClone Laboratories, Logan, UT, USA) at $37^{\circ} \mathrm{C}$ and $5 \% \mathrm{CO}_{2}$. To establish PR cell lines, the cells were incubated with $1 \mu \mathrm{M}$ paclitaxel (Sigma-Aldrich; Merck Millipore, Darmstadt, Germany) for different days. The culture medium was changed every 2 days in the presence of $1 \mu \mathrm{M}$ paclitaxel.

miR-24 mimic and miR-24 inhibitor transfection. MCF-7 and MCF-7/PR cells were transfected with miR-24 mimic or miR-24 inhibitor using Lipofectamine 2000 (Invitrogen; Thermo Fisher Scientific, Inc., Waltham, MA, USA) according to the manufacturer's protocol. A nonspecific control (Shanghai GenePharma Co., Ltd., Shanghai, China) was used for the control cells. The sequences were as follows: miR-24 mimic sense, 5'-uggcucaguucagcaggaacag-3' and antisense, 5'-guuccugcugaacugagccauu-3'; and miR-24 inhibitor, 5'-cuguuccugcugaacugagcca-3'. Upon transfection, the mRNA expression of miR-24 was measured by quantitative polymerase chain reaction (qPCR) assay.

\section{3-(4,5-dimethyl-2-thiazolyl)-2,5-diphenyl-2-H-tetrazolium-} bromide (MTT) and reverse transcription (RT)-qPCR assay. The half maximal inhibitory concentration $\left(\mathrm{IC}_{50}\right)$ of paclitaxel was calculated by cell viability using an MTT assay. After the cells were treated with $1 \mu \mathrm{M}$ paclitaxel for 60 days to induce drug resistance, $\mathrm{miR}-24 \mathrm{mimic}$ was pre-transfected into passage- 60 cells for $24 \mathrm{~h}$. Then, different concentrations of paclitaxel were added to the culture medium for another $24 \mathrm{~h}$, and the cell viability was determined as described previously (19). RT-qPCR was conducted to determine the expression level of miR-24 as previously described (20). The primers for miR-24 were those present in TaqMan $^{\mathrm{TM}}$ MicroRNA Assays (Applied Biosystems; Thermo Fisher Scientific, Inc.), and the microRNA expression was normalized to U6 small nuclear RNA (RNU6) expression according to the ${ }^{\Delta \Delta} \mathrm{Cq}$ method, as previously described (21). Other primers used were: $A B C B 9$ forward, 5'-GCTCTGGGAGAG ACCTTCCT-3' and reverse 5'-GAGCGGAAGAGACAGTTT CG-3'; glyceraldehyde 3-phosphate dehydrogenase forward, 5'-CCCACTCCTCCACCTTTGAC-3' and reverse, 5'-CAT ACCAGGAAATGAGCTTGACAA-3'; and RNU6 forward, 5'-CTCGGTTCGGCAGCACA-3' and reverse, 5'-AACGCT TCACGAATTTGCGT-3'.

Hoechst staining and western blot analysis. After transfection with miR-24 mimic for $24 \mathrm{~h}$, the MCF-7 and MCF-7/PR cells were treated with $10 \mu \mathrm{M}$ paclitaxel for another $24 \mathrm{~h}$. Then, Hoechst 33342 staining was performed as previously described (19). For western blot analysis, following different treatments, MCF-7 and MCF-7/PR cells were lysed, and western blotting was performed as previously described (19). All western blot analyses were performed $\geq 3$ times. The blots were analyzed by Quantity One v4.62 software (National Institutes of Health, Bethesda, MD, USA). Primary antibodies were all purchased from Santa Cruz Biotechnology, Inc. (Dallas, TX, USA), including anti-B-cell lymphoma (Bcl)-2 (cat. no. sc-492; dilution 1:500), anti-Bcl-2-associated X protein (Bax) (cat. no. sc-526; dilution 1:1,000), anti-cleaved caspase-3 (cat. no. sc-7148; dilution 1:1,000), anti-ABCB9 (cat. no.sc-46744; dilution $1: 1,000$ ) and anti- $\beta$-actin (cat. no. sc-1616; dilution 1:5,000).

In vitro scratch assay. MCF-7 and MCF-7/PR cells were seeded in 12-well plates and grown in complete medium. The cells were transfected with miR-24 mimic and incubated with $10 \mu \mathrm{M}$ paclitaxel. After $48 \mathrm{~h}$ of incubation, the confluent monolayer cells were scraped by pipette tips to generate a straight line scratch. Then, the wells were washed and replaced with serum-free medium in the presence of paclitaxel. The cells in the scratch were imaged under a microscope (DP70; Olympus Corporation, Tokyo, Japan) after $12 \mathrm{~h}$, and the images were used to calculate the distances between the two edges of the scratch.

Luciferase assay. The miR-24 response element (wild type or mutated) in the 3'-UTR of $A B C B 9$ was transfected into the pMIR-reporter plasmid (Ambion, Austin, TX, USA), which contained the luciferase reporter gene. MCF-7 cells were co-transfected with luciferase reporter constructs containing wild type or mutated type $A B C B 9$ 3'-UTR and miR-24 mimic in the presence/absence of miR-24 inhibitor. A luciferase assay kit (Promega Corporation, Madison, WI, USA) was used to analyze the luciferase activities according to the manufacturer's protocol.

Study population. A total of 20 human breast cancer tissues were selected from breast cancer patients that exhibited poor chemo-response after undergoing surgery at The Affiliated Cancer Hospital of Nanjing Medical University (Nanjing, China) between July 2010 and June 2014. Another group of patients $(n=20)$ with a good chemo-response was recruited as control. Tumor tissues were washed and stored in liquid nitrogen prior to the experiments. In combination with the pathological diagnosis, $>90 \%$ necrosis was considered drug-sensitive, while tumor necrosis $<90 \%$ was considered 


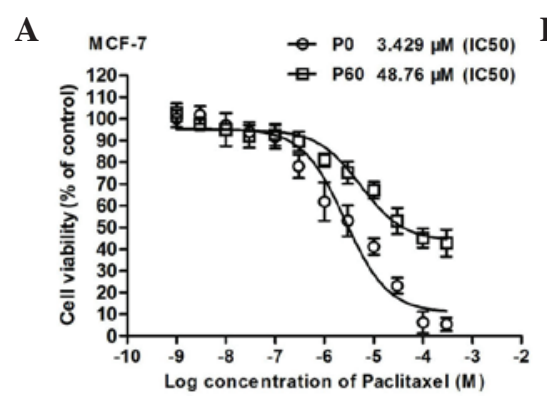

B

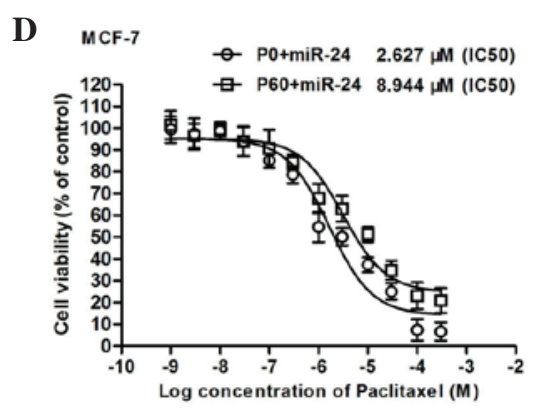

C

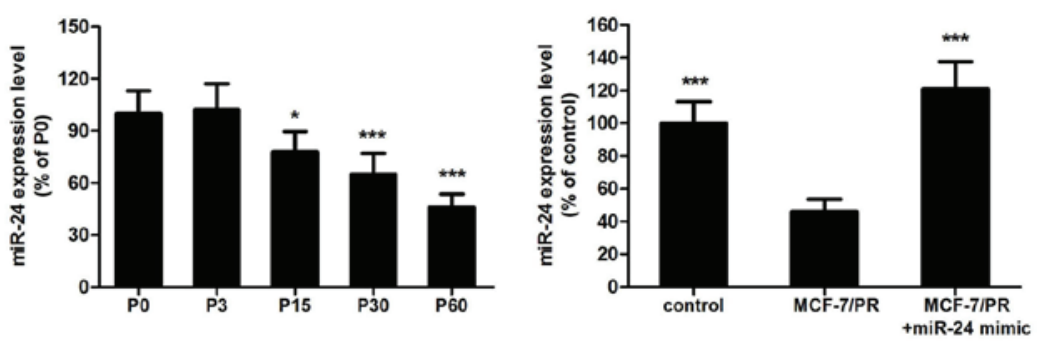

Figure 1. Overexpression of miR-24 improves the sensitivity toward paclitaxel of breast cancer cells. (A) The cytotoxicity of paclitaxel on MCF-7 (P0) and MCF-7/PR (P60) cells was determined by 3-(4,5-dimethyl-2-thiazolyl)-2,5-diphenyl-2-H-tetrazolium bromide assay (n=6). (B) Upon induction of paclitaxel resistance in MCF-7 cells for different days, the expression of miR-24 was determined by qPCR $\left({ }^{*} \mathrm{P}<0.05,{ }^{* * *} \mathrm{P}<0.001\right.$ compared with $\mathrm{P} 0$ cells, $\left.\mathrm{n}=6\right)$. $(\mathrm{C}) \mathrm{Using}$ a microRNA mimic to overexpress miR-24 in MCF-7/PR cells, the expression of miR-24 was detected by qPCR in normal MCF-7 and MCF-7/PR cells $\left({ }^{* * *} \mathrm{P}<0.001\right.$ compared with MCF-7/PR cells, $\left.\mathrm{n}=6\right)$. (D) The miR-24 mimic was added to MCF-7 or MCF-7/PR cells, and then the IC ${ }_{50}$ of paclitaxel in each cell model was determined ( $\mathrm{n}=6)$. (E) The miR-24 mimic was added to SKBR3 or SKBR3/PR cells, and then the IC ${ }_{50}$ of paclitaxel to each cell model was determined $(n=6)$. PR, paclitaxel-resistant; miR, microRNA; $\mathrm{IC}_{50}$, half maximal inhibitory concentration; qPCR, quantitative polymerase chain reaction; $\mathrm{P}$, passage.

poor responder. The total protein and total RNA from tissues were isolated using TRIzol reagent (Takara Bio Inc., Otsu, Japan), and detected by western blotting and qPCR analysis as previously described $(19,20)$. The present study was approved by the Medical Ethics Committee of Jiangsu Cancer Hospital (Nanjing, China), and written informed consent was obtained from all participants.

Animal models. A total of 40 female 8 -week-old nude BALB/c mice (20-22 g) were purchased from the Model Animal Research Center of Nanjing University (Nanjing, China). Mice were maintained at $25^{\circ} \mathrm{C}$ and exposed to $12 \mathrm{~h} \mathrm{light/dark} \mathrm{cycles} \mathrm{with} \mathrm{free} \mathrm{access} \mathrm{to} \mathrm{food} \mathrm{and} \mathrm{water.}$ Prior to the experiment, mice were allowed to acclimate for 5 days. A cell suspension of MCF-7, MCF-7/PR or miR-24 mimic-pretreated MCF-7/PR cells was prepared $\left(10^{8}\right.$ cells $\left./ \mathrm{ml}\right)$. Then, the cell suspension was subcutaneously injected into the right upper flank of the 40 nude mice. When tumors mass reached $50 \mathrm{~mm}$ in diameter, $10 \mathrm{mg} / \mathrm{kg}$ paclitaxel was intraperitoneally injected once every 2 days. Tumor volume was recorded and calculated as $\left(\mathrm{r}^{2} \mathrm{xl}\right) / 2$, where $\mathrm{r}$ and 1 are, respectively, the short and long diameter of the tumors. After 14 days of administration, the animals were sacrificed and tumor tissue was removed. All animal studies were carried out under the supervision of the Committee for Animal Experiments of Nanjing Medical University (Nanjing, China).

Terminal deoxynucleotidyl transferase-mediated dUTP nick end labeling (TUNEL) staining. Tumor tissues were fixed in $4 \%$ paraformaldehyde for paraffin sections. Then, a TUNEL kit (Roche Diagnostics, Indianapolis, IN, USA) was used to stain the apoptotic cells according to the manufacturer's protocol. The apoptotic ratio was calculated as the number of TUNEL-positive cells divided the by total number of cells.

Statistical analysis. Data were expressed as the mean \pm standard deviation. All statistical analysis was performed using PASW Statistics 18.0 software (SPSS, Inc., Chicago, IL, USA). Statistical comparisons between groups were analyzed using the $t$ test and one-way analysis of variance. $\mathrm{P}<0.05$ was considered to indicate a statistically significant difference.

\section{Results}

Overexpression of miR-24 increases the sensitivity to paclitaxel of breast cancer cell lines. After the MCF-7 breast cancer cells were treated for 60 days with $1 \mu \mathrm{M}$ paclitaxel, the $\mathrm{IC}_{50}$ of paclitaxel in MCF-7/PR cells increased by 16 -fold compared with that in normal MCF-7 cells (Fig. 1A). In this PR cell line, the expression of miR-24 decreased in a time-dependent manner (Fig. 1B). Thus, to further investigate the effect of miR-24 on the cytotoxicity of paclitaxel, a microRNA mimic was used to overexpress miR-24 in MCF-7 (Fig. 1C) and other breast cancer cell lines (data not shown). It was observed that overexpression of miR-24 did not affect the $\mathrm{IC}_{50}$ of paclitaxel in normal MCF-7 cells $\left(\mathrm{IC}_{50}\right.$ changed from 2.627 to $3.429 \mu \mathrm{M}$, Fig. 1D). On the contrary, using a microRNA mimic to increase the miR-24 expression level in MCF-7/PR cells could obviously improve the sensitivity toward paclitaxel of this drug-resistant cell model $\left(\mathrm{IC}_{50}\right.$ changed from 8.944 to $48.760 \mu \mathrm{M}$, Fig. 1D). Similar results were also observed in SKBR3 breast cancer cells (Fig. 1E and F). 
A
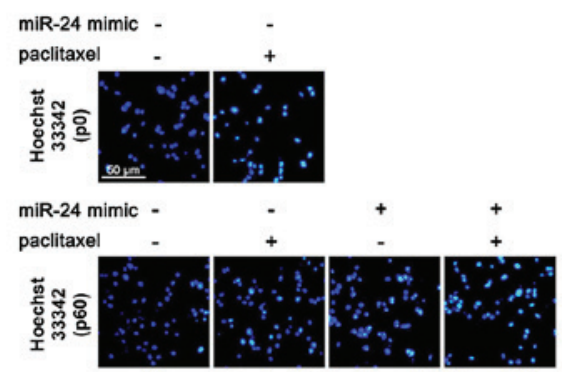

D

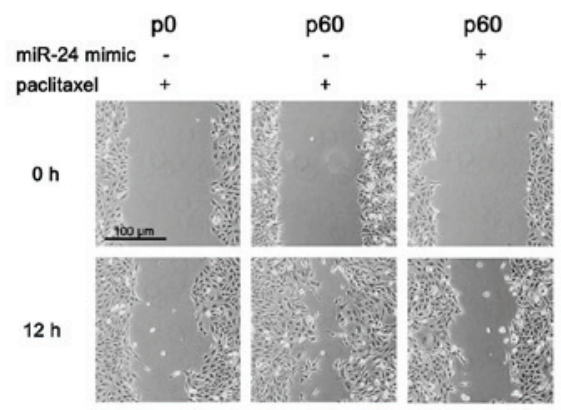

G

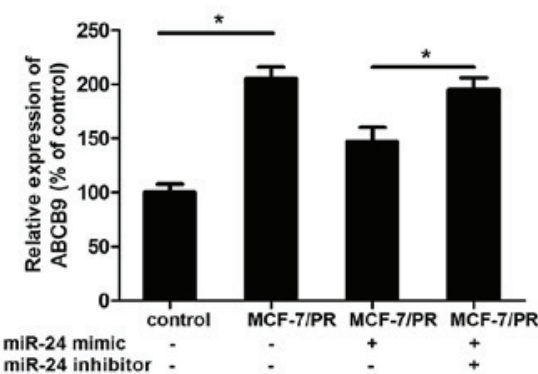

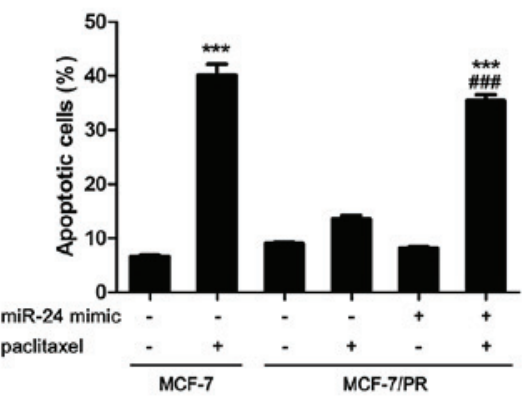

C

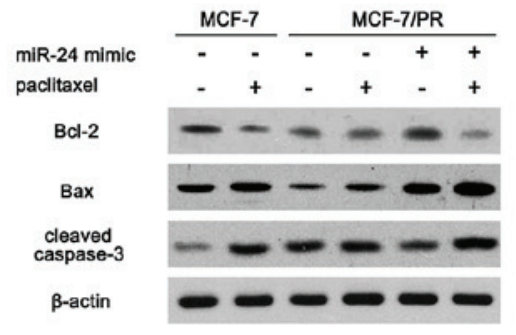

E

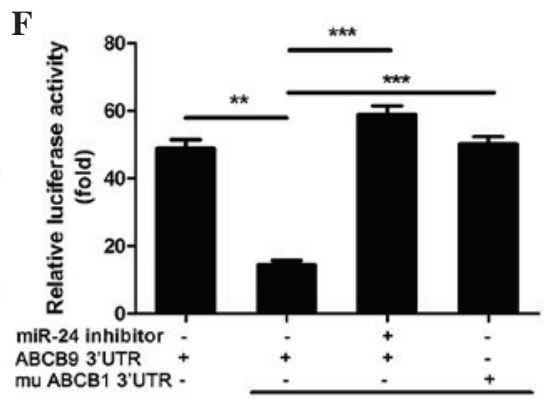

$\mathbf{H}$

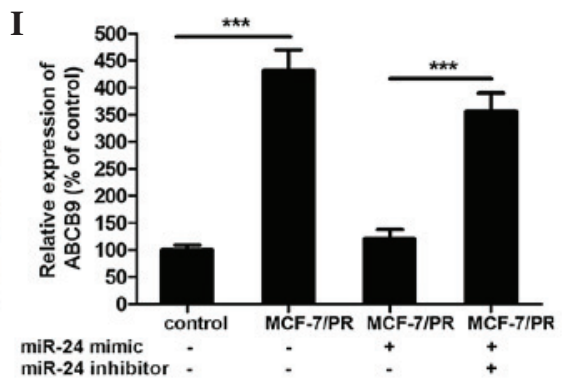

Figure 2. Overexpression of miR-24 increases paclitaxel-induced apoptosis and inhibition of migration in MCF-7/PR cells via regulation of ABCB9 expression. (A) Hoechst 33342 staining was used to mark apoptotic cells, and images were recorded by fluorescence microscopy (scale bar=50 $\mu$ m). (B) The apoptoticpositive cells were counted and divided by the total cell number to calculate the apoptotic ratio. In total, 15 random images from six parallel wells were selected

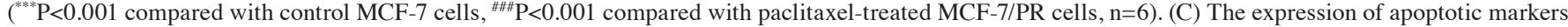
in different groups was determined by western blotting. $\beta$-actin was used as internal reference. All blots were repeated $\geq 3$ times. (D) The migration of cells was investigated by scratch test (scale bar $=100 \mu \mathrm{m}$ ). (E) Prediction of the miR-24 binding site on ABCB9 and ABCB9 mutation site. (F) Luciferase assay was performed to detect whether miR-24 could directly binding to the 3 '-UTR region of ABCB9 $\left(^{* *} \mathrm{P}<0.01,{ }^{* * * *} \mathrm{P}<0.001, \mathrm{n}=6\right)$. (G) The messenger RNA expression of ABCB9 was determined by quantitative polymerase chain reaction $\left({ }^{*} \mathrm{P}<0.01, \mathrm{n}=6\right)$. $(\mathrm{H})$ The protein expression of $\mathrm{ABCB} 9$ was determined by western blotting. (I) The blot was analyzed by optical density (*** $\mathrm{P}<0.001, \mathrm{n}=3$ ). PR, paclitaxel-resistant; miR, microRNA; Bcl-2, B-cell lymphoma-2; Bax, Bcl-2-associated X protein; ABC, ATP-binding cassette; mu, mutant; UTR, untranslated region.

Overexpression of miR-24 increases paclitaxel-induced apoptosis and inhibition of migration in MCF-7/PR cells via regulation of $A B C B 9$ expression. As miR-24 could increase the cytotoxic effect of paclitaxel on breast cancer cells, the present study further investigated the effect of miR-24 on paclitaxel-induced cell apoptosis and inhibition of migration. Using Hoechst 33342 staining, paclitaxel-induced apoptosis was determined. The apoptotic cells displayed highly bright and shrunk nuclei (Fig. 2A). The apoptotic rate in $10 \mu \mathrm{M}$ paclitaxel-treated MCF-7 cells reached $40 \%$, which was 4-fold higher than that in control cells (Fig. 2B). However, this cytotoxic effect was not obvious in MCF-7/PR cells (Fig. 2B). Using a miR-24 mimic, the cytotoxicity of $10 \mu \mathrm{M}$ paclitaxel on MCF-7/PR cells was partially recovered (Fig. 2B). Furthermore, it was noticed that $10 \mu \mathrm{M}$ paclitaxel could reduce the expression of the anti-apoptotic protein Bcl-2, increase the expression of the apoptotic marker Bax and activate caspase-3 in normal MCF-7 cells, while these effects were suppressed in MCF-7/PR cells (Fig. 2C). However, increased miR-24 expression level restored the pro-apoptotic effect of paclitaxel on drug-resistant cells (Fig. 2C). In addition, the effect of miR-24 on the paclitaxel-regulated migration of MCF-7 cells was also detected. Scratch test revealed that the inhibitory effect of $10 \mu \mathrm{M}$ paclitaxel on the migration of MCF-7/PR cells was not as obvious as that observed in normal MCF-7 cells (Fig. 2C). However, in accordance with the results of the apoptotic experiments, overexpression of miR-24 improved the inhibitory effect of paclitaxel on the migration of MCF-7/PR cells (Fig. 2D).

The above results demonstrated that upregulating miR-24 expression did not affect normal MCF-7 cells survival (Fig. 1D). Thus, it was speculated that miR-24 may increase the sensitivity to paclitaxel of MCF-7/PR cells through regulating efflux pumps or reducing the metabolism of the compound. Using online microRNA target prediction tools [including TargetScanHuman 6.2 (http://www.targetscan.org/vert_71/), miRBase (http://www.mirbase.org/) and starBase (http://starbase.sysu.edu.cn/)], it was predicted that the 3'-UTR region of 
A

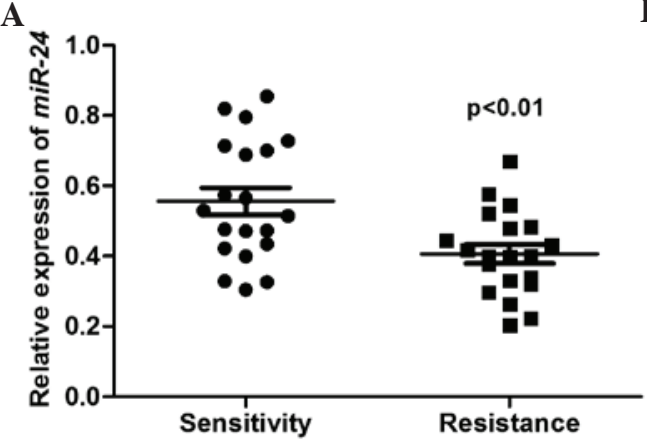

C

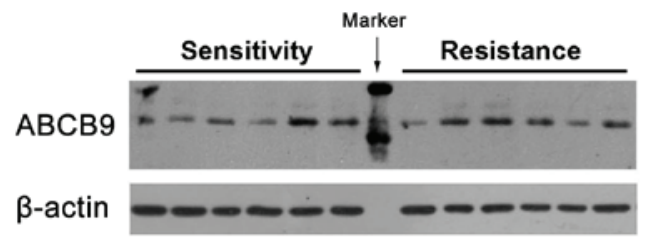

D
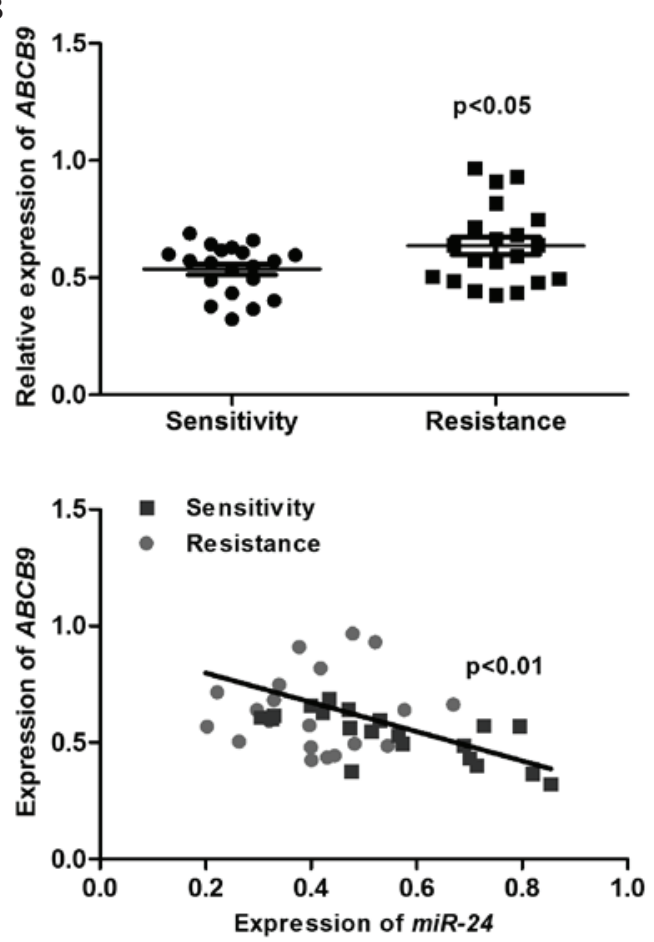

Figure 3. Expression of miR-24 and ABCB9 in breast cancer patients. (A and B) The expression of (A) miR-24 and (B) ABCB9 was detected by quantitative polymerase chain reaction in drug-resistant and drug-sensitive breast cancer patients $(n=20)$. (C) The expression of $A B C B 9$ was detected by western blotting in drug-resistant and drug-sensitive breast cancer patients (six samples were analyzed in each group). (D) Correlation analysis was performed between the expression level of miR-24 and the $A B C B 9$ gene in breast cancer patients. miR, microRNA; ABC, ATP-binding cassette.

A
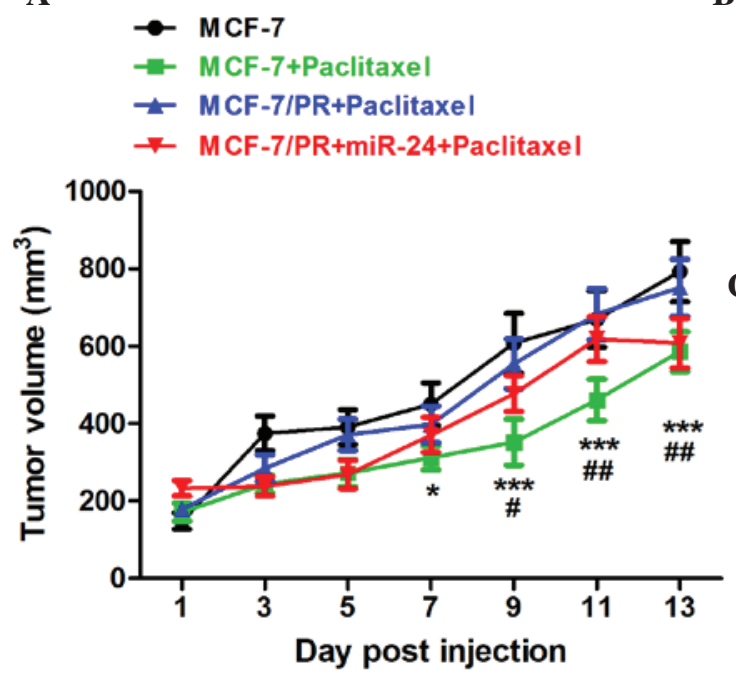

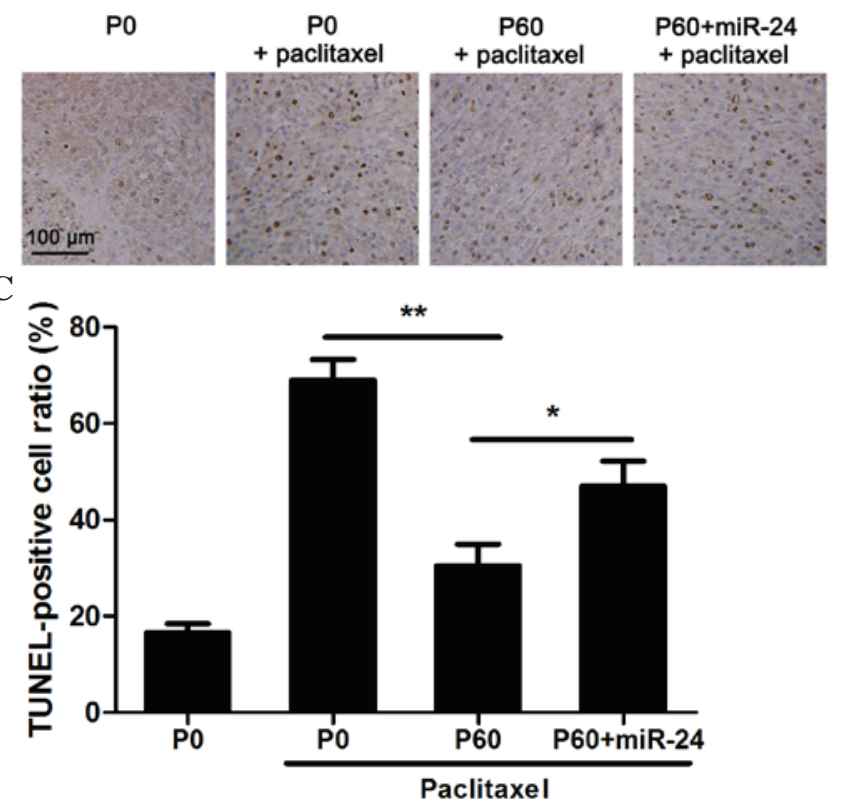

Figure 4. Effect of paclitaxel on miR-24-overexpressing MCF-7/PR cells in implanted nude mice. (A) Tumor growth in nude mice was determined every 2 days. ${ }^{*} \mathrm{P}<0.05,{ }^{* * * *} \mathrm{P}<0.001$ in the MCF-7/PR-miR-24 group treated with paclitaxel compared with the control MCF-7 group; ${ }^{*} \mathrm{P}<0.05,{ }^{\# \#} \mathrm{P}<0.01$ in the MCF-7/PR-miR-24 group treated with paclitaxel compared with the MCF-7/PR group treated with paclitaxel, $n=8$. (B) TUNEL assay was performed to observe the apoptotic cells. Apoptotic nuclei exhibited brown staining, while all nuclei were stained by hematoxylin with blue dye. Scale bar=100 $\mu \mathrm{m}$. (C) The apoptotic ratio was calculated as the number of TUNEL-positive cells divided by the total cell number. ${ }^{*} \mathrm{P}<0.05,{ }^{* *} \mathrm{P}<0.01, \mathrm{n}=8$. PR, paclitaxel-resistant; miR, microRNA; $\mathrm{P}$, passage; TUNEL, terminal deoxynucleotidyl transferase-mediated dUTP nick end labeling.

ABCB9 has possible binding positions for miR-24 (Fig. 2E). To confirm this concept, a miR-24 mimic and a ABCB9 3'-UTR wild type or mutated luciferase reporter were co-transfected into MCF-7/PR cells. It was observed that the miR-24 mimic decreased the luciferase activity of ABCB9 3'-UTR wild type-transfected cells, but not that of cells transfected with the mutated version (Fig. 2F). In addition, using a designed miR-24 inhibitor, ABCB9 3'-UTR wild type yielded similar results 
compared with the mutated version (Fig. 2F). The expression of $\mathrm{ABCB} 9$ was next determined at both the mRNA and protein level by qPCR and western blot analysis, respectively. It was observed that the expression of ABCB9 increased significantly in MCF-7/PR cells in comparison with that in normal MCF-7 cells, but miR-24 mimic reduced ABCB9 expression at both the mRNA and protein level (Fig. 2F-H). Meanwhile, miR-24 inhibitor could abolish this effect.

Expression of $m i R-24$ and $A B C B 9$ in drug-resistant breast cancer patients. As miR-24 and ABCB9 are important in paclitaxel resistance of breast cancer cells, the expression level of miR-24 and ABCB9 in PR breast cancer patients was examined. Fig. 3A indicates that the miR-24 level was significantly decreased in tumor tissues from breast cancer patients with poor response to paclitaxel compared with that observed in tumor tissues from patients with good drug sensitivity. On the contrary, the expression of ABCB9 in the drug-resistant group was significantly higher than that in patients with good drug sensitivity (Fig. 3B). This result was also verified by western blotting (Fig. 3C). Furthermore, correlation analysis revealed that the expression of miR-24 was negatively correlated with the expression of ABCB9 (Fig. 3D), which supported the evidence that miR-24 downregulated the expression of ABCB9, thereby increasing drug sensitivity.

Overexpression of miR-24 increases the sensitivity of paclitaxel to MCF-7/PR cells in vivo. The effect of miR-24 on the sensitivity toward paclitaxel of MCF-7/PR cells in vivo was next investigated using a nude mouse model. Using a miR-24 mimic, a high expression level of miR-24 could be sustained for $>2$ weeks (data not shown). It was observed that $10 \mathrm{mg} / \mathrm{kg}$ paclitaxel could significantly reduce tumor growth in MCF-7-implanted nude mice (Fig. 4A). However, paclitaxel at this dose was not able to affect the growth of tumors derived from MCF-7/PR cells (Fig. 4A). On the contrary, when MCF-7/PR cells were pre-transfected with a miR-24 mimic, the anti-tumor effect of $10 \mathrm{mg} / \mathrm{kg}$ paclitaxel was partially recovered (Fig. 4A). Apoptosis in tumor tissues was detected by TUNEL assay. Following 2 weeks of treatment, $10 \mathrm{mg} / \mathrm{kg}$ paclitaxel could markedly induce tumor cell apoptosis (Fig. 4B and C). In accordance with the results of the experiment performed in vitro, paclitaxel induced less apoptosis in MCF-7/PR cell-implanted tumor tissue than that in the tissues of normal MCF-7 cell-implanted nude mice (Fig. 4B and C). Furthermore, pre-transfection with the miR-24 mimic could recover the pro-apoptotic effect of paclitaxel in vivo.

\section{Discussion}

Drug resistance in breast cancer has been regarded as one of the primary obstacles contributing to chemotherapy failure (22). Thus, it is necessary to explore novel therapeutic methods to reduce drug resistance for the treatment of breast cancer. In the present study, it was observed that the expression of miR-24 was significantly reduced in tumor tissues from PR breast cancer patients and PR breast cancer cell lines. Further in vitro experiments confirmed that upregulating miR-24 expression could partially recover the sensitivity to paclitaxel of breast cancer cells. Therefore, miR-24 may be a useful target to reduce drug resistance and a possible marker to guide clinical medication.
miR-24 has been reported to be an oncogene in oral carcinoma (23), prostate cancer (24) and lung cancer (25). In addition, miR-24 could promote cell proliferation in lung cancer (25) and hepatocellular carcinoma (26). However, miR-24 could inhibit cell proliferation in gastric cancer $(27,28)$, and acts as a tumor suppressor in colon cancer (29). In breast cancer, the role of miR-24 also remains controversial. The expression of miR-24 in serum from early breast cancer patients was decreased in a previous study (30), but another study using paraffin-embedded tissues observed that the expression of miR-24 was upregulated (31). In addition, Du et al reported that the expression of miR-24 was higher in breast cancer samples than that in benign breast tissues, and speculated that miR-24 may enhance tumor invasion (32). Therefore, as an important microRNA associated with cancer development, the role of miR-24 in breast and other cancers should be further investigated.

Using microRNA target prediction tools, the present authors identified that ABCB9 could be one of the target genes of miR-24. ABCB9 is a member of the $\mathrm{ABC}$ sub-family $B$, which has been reported to be involved in drug intracellular trafficking, thereby affecting chemotherapy-related multidrug resistance (MDR) $(33,34)$. Thus, it was speculated that miR-24 may affect the expression of ABCB9 to regulate the sensitivity toward paclitaxel of breast cancer cells. It was identified that miR-24 could directly bind to the 3'-UTR of ABCB9, thereby inhibiting the translation of $\mathrm{ABCB}$ 9. These results were in accordance with those from previous studies $(35,36)$. Overall, the above results indicated that downregulation of $\mathrm{ABCB} 9$ may contribute to the effect of miR-24 on the sensitivity toward paclitaxel of breast cancer cells. Furthermore, the present study confirmed that ABCB9 was also upregulated in chemotherapyresistant breast cancer patients, which was negatively correlated with the level of miR-24, suggesting that miR-24 was associated with drug resistance in breast cancer via regulating $\mathrm{ABCB} 9$ expression. Unlike other members of the $\mathrm{ABC}$ transporter protein family, the regulatory effect of $\mathrm{ABCB} 9$ on drug resistance has not been frequently reported $(34,36)$. The present study provided novel evidence regarding the role of ABCB9 in chemotherapy-related MDR. Furthermore, the role of miR-24 in PR breast cancer cells was evaluated in vivo using a nude mouse model. It was observed that miR-24 increased the sensitivity to paclitaxel of MCF-7/PR-implanted nude mice. Although multiple mechanisms may be involved, including $\mathrm{p} 450$-related drug metabolism and various $\beta$-tubulin mutations, which require further investigation in miR-24associated drug resistance, the present results suggested that miR-24 could be important in drug resistance of breast cancer cells, and could improve the sensitivity toward paclitaxel both in vitro and in vivo.

In conclusion, the present study demonstrated for the first time that miR-24 improves the sensitivity toward paclitaxel of breast cancer cells, suggested an important novel function for miR-24, and provided a possible microRNA target and a useful marker in the clinical treatment of breast cancer.

\section{Acknowledgements}

The present study was supported by a grant from Jiangsu Cancer Hospital (Nanjing, China; grant no. ZM201105). 


\section{References}

1. Bottos A and Hynes NE: Cancer: Staying together on the road to metastasis. Nature 514: 309-310, 2014.

2. Xuan Q, Ji H, Tao X, Xu Y and Zhang Q: Quantitative assessment of HER2 amplification in HER2-positive breast cancer: Its association with clinical outcomes. Breast Cancer Res Treat 150: $581-588,2015$

3. Wang Y, Zhang Y, Pan C, Ma F and Zhang S: Prediction of poor prognosis in breast cancer patients based on microRNA-21 expression: A meta-analysis. PLoS One 10: e0118647, 2015.

4. Grobosch T, Schwarze B, Stoecklein D and Binscheck T: Fatal poisoning with Taxus baccata: Quantification of paclitaxel (taxol A), 10-deacetyltaxol, baccatin III, 10-deacetylbaccatin III, cephalomannine (taxol B), and 3,5-dimethoxyphenol in body fluids by liquid chromatography-tandem mass spectrometry. J Anal Toxicol 36: 36-43, 2012.

5. Johnson MT, Reichley R, Hoppe-Bauer J, Dunne WM, Micek S and Kollef M: Impact of previous antibiotic therapy on outcome of Gram-negative severe sepsis. Crit Care Med 39: 1859-1865, 2011.

6. Ganoth A, Merimi KC and Peer D: Overcoming multidrug resistance with nanomedicines. Expert Opin Drug Deliv 12: 223-238, 2015.

7. Ohashi-Kobayashi A, Ohashi K, Du WB, Omote H, Nakamoto R, Al-Shawi $\mathrm{M}$ and Maeda M: Examination of drug resistance activity of human TAP-like (ABCB9) expressed in yeast. Biochem Biophys Res Commun 343: 597-601,2006.

8. Kaur P, Garg T, Rath G, Murthy RS and Goyal AK: Development, optimization and evaluation of surfactant-based pulmonary nanolipid carrier system of paclitaxel for the management of drug resistance lung cancer using Box-Behnken design. Drug Deliv 23: 1912-1925,2016.

9. Zhu Z, Mu Y, Qi C, Wang J, Xi G, Guo J, Mi R and Zhao F: CYP1B1 enhances the resistance of epithelial ovarian cancer cells to paclitaxel in vivo and in vitro. Int J Mol Med 35: 340-348, 2015.

10. Verma $\mathrm{K}$ and Ramanathan K: Investigation of paclitaxel resistant $\mathrm{R} 306 \mathrm{C}$ mutation in $\beta$-tubulin-a computational approach. J Cell Biochem 116: 1318-1324, 2015.

11. Png KJ, Halberg N, Yoshida M and Tavazoie SF: A microRNA regulon that mediates endothelial recruitment and metastasis by cancer cells. Nature 481: 190-194, 2011.

12. Shibayama Y, Kondo T, Ohya H, Fujisawa SI, Teshima T and Iseki K: Upregulation of microRNA-126-5p is associated with drug resistance to cytarabine and poor prognosis in AML patients. Oncol Rep 33: 2176-2182, 2015.

13. Acunzo M, Romano G, Wernicke D and Croce CM: MicroRNA and cancer-a brief overview. Adv Biol Regul 57: 1-9, 2015.

14. Yu PN, Yan MD, Lai HC, Huang RL, Chou YC, Lin WC, Yeh LT and Lin YW: Downregulation of miR-29 contributes to cisplatin resistance of ovarian cancer cells. Int J Cancer 134 542-551, 2014

15. Zhang Y, Duan G and Feng S: MicroRNA-301a modulates doxorubicin resistance in osteosarcoma cells by targeting AMP-activated protein kinase alpha 1 . Biochem Biophys Res Commun 459: 367-373, 2015.

16. Chatterjee A, Chattopadhyay D and Chakrabarti G: miR-17-5p downregulation contributes to paclitaxel resistance of lung cancer cells through altering beclin1 expression. PLoS One 9: e95716, 2014.

17. Gao Y, Liu Y, Du L, Li J, Qu A, Zhang X, Wang L and Wang C: Downregulation of miR-24-3p in colorectal cancer is associated with malignant behavior. Med Oncol 32: 362, 2015.

18. Guo C, Deng Y, Liu J and Qian L: Cardiomyocyte-specific role of miR-24 in promoting cell survival. J Cell Mol Med 19: 103-112, 2015
19. Wang T, Sun P, Chen L, Huang Q, Chen K, Jia Q, Li Y and Wang H: Cinnamtannin D-1 protects pancreatic $\beta$-cells from palmitic acid-induced apoptosis by attenuating oxidative stress. J Agric Food Chem 62: 5038-5045, 2014.

20. Wu J, Sun P, Zhang X, Liu H, Jiang H, Zhu W and Wang $H$ Inhibition of GPR40 protects MIN6 $\beta$ cells from palmitateinduced ER stress and apoptosis. J Cell Biochem 113: 1152-1158, 2012.

21. Wang XW, Xi XQ, Wu J, Wan YY, Hui HX and Cao XF: microRNA-206 attenuates tumor proliferation and migration involving the downregulation of NOTCH3 in colorectal cancer. Oncol Rep 33: 1402-1410, 2015.

22. Huang J, Li H and Ren G: Epithelial-mesenchymal transition and drug resistance in breast cancer (Review). Int J Oncol 47: 840-848, 2015

23. Lin SC, Liu CJ, Lin JA, Chiang WF, Hung PS and Chang KW: miR-24 up-regulation in oral carcinoma: Positive association from clinical and in vitro analysis. Oral Oncol 46: 204-208, 2010

24. Qin W, Shi Y, Zhao B, Yao C, Jin L, Ma J and Jin Y: miR-24 regulates apoptosis by targeting the open reading frame (ORF) region of FAF1 in cancer cells. PLoS One 5: e9429, 2010

25. Zhao G, Liu L, Zhao T, Jin S, Jiang S, Cao S, Han J, Xin Y, Dong Q, Liu X and Cui J: Upregulation of miR-24 promotes cell proliferation by targeting NAIF1 in non-small cell lung cancer. Tumour Biol 36: 3693-3701, 2015.

26. Liu YX, Long XD, Xi ZF, Ma Y, Huang XY, Yao JG, Wang C, Xing TY and Xia Q: MicroRNA-24 modulates aflatoxin B1-related hepatocellular carcinoma prognosis and tumorigenesis. Biomed Res Int 2014: 482926, 2014.

27. Duan Y, Hu L, Liu B, Yu B, Li J, Yan M, Yu Y, Li C, Su L, Zhu Z, et al: Tumor suppressor miR-24 restrains gastric cancer progression by downregulating RegIV. Mol Cancer 13: 127, 2014.

28. Wu J, Zhang YC, Suo WH, Liu XB, Shen WW, Tian H and Fu GH: Induction of anion exchanger-1 translation and its opposite roles in the carcinogenesis of gastric cancer cells and differentiation of K562 cells. Oncogene 29: 1987-1996, 2010.

29. Mishra PJ, Song B, Mishra PJ, Wang Y, Humeniuk R, Banerjee D, Merlino G, Ju J and Bertino JR: MiR-24 tumor suppressor activity is regulated independent of p53 and through a target site polymorphism. PLoS One 4: e8445, 2009.

30. Sochor M, Basova P, Pesta M, Dusilkova N, Bartos J, Burda P, Pospisil V and Stopka T: Oncogenic microRNAs: miR-155, miR-19a, miR-181b and miR-24 enable monitoring of early breast cancer in serum. BMC Cancer 14: 448, 2014.

31. Yin JY, Deng ZQ, Liu FQ, Qian J, Lin J, Tang Q, Wen XM, Zhou JD, Zhang YY and Zhu XW: Association between mir-24 and mir-378 in formalin-fixed paraffin-embedded tissues of breast cancer. Int J Clin Exp Pathol 7: 4261-4267, 2014.

32. Du WW, Fang L, Li M, Yang X, Liang Y, Peng C, Qian W, O'Malley YQ, Askeland RW, Sugg SL, et al: MicroRNA miR-24 enhances tumor invasion and metastasis by targeting PTPN9 and PTPRF to promote EGF signaling. J Cell Sci 126: 1440-1453, 2013.

33. Demirel O, Bangert I, Tampé R and Abele R: Tuning the cellular trafficking of the lysosomal peptide transporter TAPL by its N-terminal domain. Traffic 11: 383-393, 2010.

34. Hlavata I, Mohelnikova-Duchonova B, Vaclavikova R, Liska V, Pitule P, Novak P, Bruha J, Vycital O, Holubec L, Treska V, et al: The role of $\mathrm{ABC}$ transporters in progression and clinical outcome of colorectal cancer. Mutagenesis 27: 187-196, 2012

35. Xu F, Wang F, Yang T, Sheng Y, Zhong T and Chen Y: Differential drug resistance acquisition to doxorubicin and paclitaxel in breast cancer cells. Cancer Cell Int 14: 538, 2014.

36. Dong Z, Zhong Z, Yang L, Wang S and Gong Z: MicroRNA-31 inhibits cisplatin-induced apoptosis in non-small cell lung cancer cells by regulating the drug transporter ABCB9. Cancer Lett 343: 249-257, 2014. 